\title{
Cloud Storage Optimization Analysis with Energy Consumption and Life Cycle
}

\author{
Li Yonghong ${ }^{1}$, Zhou $\mathrm{Na}^{2}$ and Zhao Guofeng ${ }^{2}$ \\ ${ }^{1}$ Earthquake Administration of Shandong Province, Jinan 250014, China \\ ${ }^{2}$ China Earthquake Networks Center, Beijing 100045, China \\ eqwww@163.com
}

\begin{abstract}
With the development of network and storage technology, providing a shareable and convenient cloud storage service has become a major new application mode and development trend of internet, however, the system scale is huge and storage content is variable, so traditional performance evaluation and testing method are no suitable for cloud storage platform. This paper provides the thinking of performance evaluation of cloud storage system, sets up multiple-index $3 D$ evaluation system facing different users and adaptable to different stages. It applies cloud testing with cloud scheme to test node visiting performance in different physical positions.
\end{abstract}

Keywords: Cloud storage; Performance evaluation; Cloud test loud; Optimize

\section{Introduction}

With the development of network and storage technology, providing shareable and convenient network cloud storage service has become a new type of application model and development trend of internet. The wide application of cloud computing and cloud storage raises huge challenge to the existed storage technology in systematic structure, performance, dynamic setting configure, interactive mode, reliability and safety, etc., The technological level and ability has strategic significance on national digital infrastructure construction and information security. Whether various applications and network storage constructed on the platform of cloud storage will be successful or not depends on the performance provided by storage service. Different from traditional storage service, the object of cloud storage is uncertain public and access scale, access focus and access mode have been changed; on the other hand, the acquisition bottleneck of storage resource is not I/O but public network. Therefore, the performance simulation and evaluation technology of traditional storage system and distributed file system cannot be adaptable to cloud storage service totally.

\section{Cloud Storage Evaluation}

Cloud storage is mainly to increase multiple access interfaces and application methods on the basis of distributed file system and data management in order to achieve elastic management method of allocation as demand. Its multiple core technologies originate from mass storage system. General composition of cloud storage is as in shown in Diagram 1.Compared with evaluation technology of traditional high-performance computing and high-end fault-tolerant computer mass storage system, cloud storage evaluation and simulation technology have the following characteristics:

\subsection{Various Roles Concern about Different Aspects}

Cloud storage system has many related roles and different roles have different concerns to cloud storage system. 


\subsection{Evaluation and Simulation of System Level}

Cloud storage system has big scale and wide distribution. It involves many data management technologies and strategies, so it's difficult to evaluate the systematic performance and reliability.

\subsection{Distributed and Multiple-User Load Simulation}

Parallel scale of actual system users exceeds load generative ability of lab. User's performance in different physical location has greater differences.

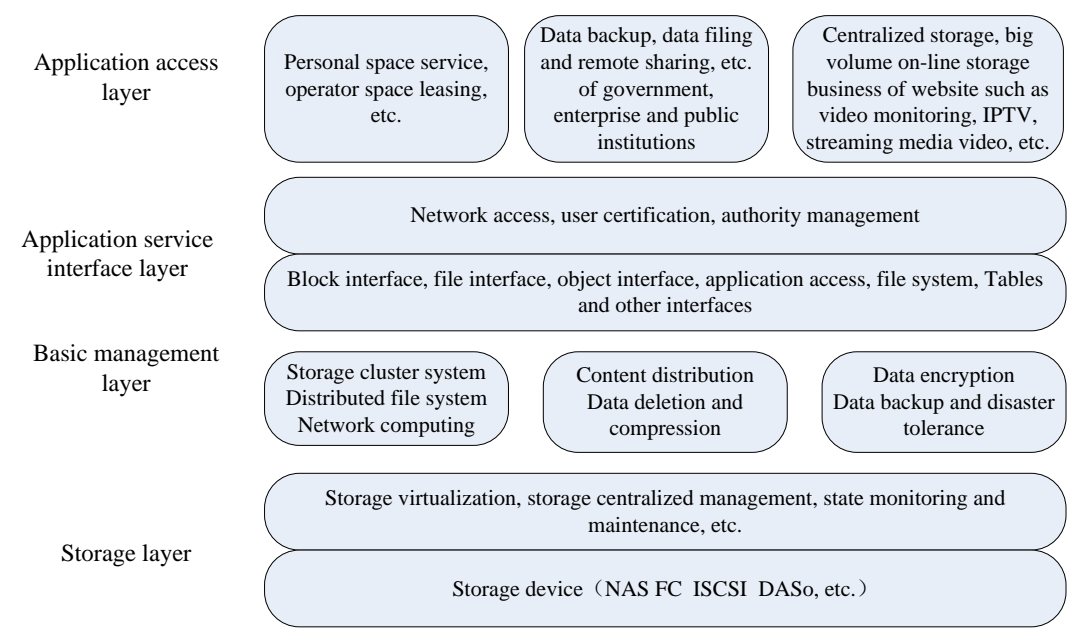

Diagram 1. Basic Components of Cloud Storage System

The research on cloud storage service performance has become a hot topic of cloud storage and cloud computing in academic circle. Garfinkel Error! Reference source not found. and Hill Error! Reference source not found. et. al., test the performance of Amazon AWS and Windows Azure respectively, such as access speed of database, network throughput and availability, etc. In addition, there are some researches making comparison of storage performance of traditional storage system and Amazon S3 through traditional testing tool, for example, Simson L. Garfinkel Error! Reference source not found. et. al., from Harvard University make test according to EC2 (Elastic Compute Cloud), S3 (Simple Storage Service) and SQS (Simple Queue Service) of AWS. They only evaluate performance and availability of object storage. YCSB Error! Reference source not found. is developed by Yahoo Company, which mainly aims at the evaluation of key/value cloud storage system. Cloudstone Error! Reference source not found. is a program similar to YCSB. It's a tool set developed by UC Berkeley and SUN Microsystems jointly. The purpose is to provide a benchmark based on social networking service application. Filebench Error! Reference source not found. is an automatic testing tool of file system performance. It tests the performance of file system through simulating the load of real application server rapidly.

The current performance evaluation index is designed from task angle and compared from higher application layers. Network environment and application mode of users are different, so these test indexes don't have comparability. In addition, these indexes cannot be used for performance evaluation of system level nor analyze the performance bottleneck of system.

\section{Cloud Storage Performance Evaluation Thinking}

\subsection{Cloud Storage Evaluation System}


Facing different users, cloud storage evaluation system is adaptable to multi-index comprehensive evaluation system at different stages. Evaluation system includes evaluation index, evaluation service object and service life cycle. Evaluation index includes performance, efficiency, elasticity, availability and manageability, etc. Evaluation service object includes final user, cloud computing service provider, cloud computing infrastructure provider and equipment provider, etc. Service life cycle covers the $\mathrm{R} \& \mathrm{D}$, operation and dilatancy of cloud computing and cloud storage system.

\subsection{Cloud Storage Evaluation Environment}

The basic thought of evaluation is applying parallel evaluation and comprehensive analysis method to get performance index of system. The whole evaluation system is made up of the following parts:

3.2.1. Basic Storage Device Test: For basic storage device test, load generative software produces mass of load to test throughput rate and IOPS performance of cloud storage device.

3.2.2. Virtual Storage Pool Test: It connects multiple storage devices together through high-speed network, forms storage pool of cloud storage system and then distributes it to each test node. Every node is installed with load generative software used for testing.

3.2.3. Interface Application Test: Make test for system interface application.

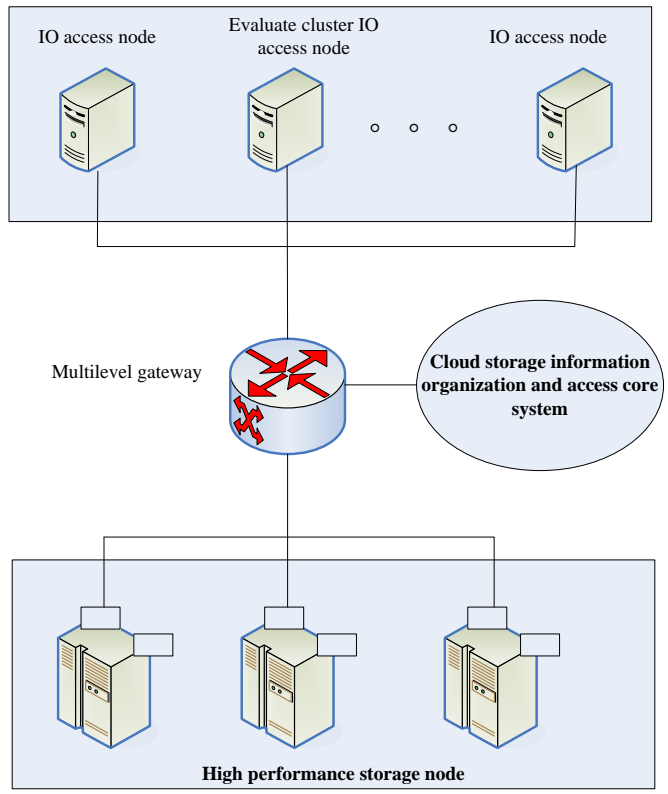

Diagram 2. Cloud Storage System Evaluation Environment Schematic

\subsection{Cloud Storage Evaluation Content}

For evaluation content, it stresses starting from these three dimensions of benchmark index test, different roles of users and different stages of research and operation of cloud storage system:

On the basis of traditional mass storage system index, benchmark index test combines the feature of cloud storage system (that is supply as needed, rapid elasticity configuration, big access scale and wide distribution; high parallel/high throughput, balance of computing and IO demand, large total quantity and rapid expansion; and international construction features of mass resources convergence, stress high- 
performance density, high-performance power consumption, etc.) and proposes purposeful new index and testing method and tool, including benchmark test in elasticity shortening environment and various indexes in high parallel/high connection situation, related tests to virtualization, performance per volume, performance cost and throughput rate/ power consumption, etc.

Cloud storage system provides access, usage, development, configuration and management interface of different levels/ authorities for final users, application developer and system operator. Cloud storage is a kind of service mode of IT resource provided as needed in nature, so it should provide certain service level agreement for different roles of users. There is an important focus of SLA, namely "measurability" and "measuring method". We propose to carry out proper research on index computing method and measuring method for different types of SLA measuring indexes.

It achieves the purpose of promoting construction with evaluation through the circulation of evaluation-->feedback-->improvement-->evaluation and provides service for successful research of each core system of cloud storage. Meanwhile, cloud storage system stress providing IT service outward, so it has the feature of updating constantly according to operation feedback situation (for example, Google has so-called "permanent Beta version" network service development and on-line strategy ). It needs acquiring all kinds of situations of operation from system monitoring interface through on-line testing method in system operation and maintenance period. Through various data mining methods, it evaluates system state so as to help system maintainer and developer to locate possible problems and bottleneck and improve the basis of updating and improvement.

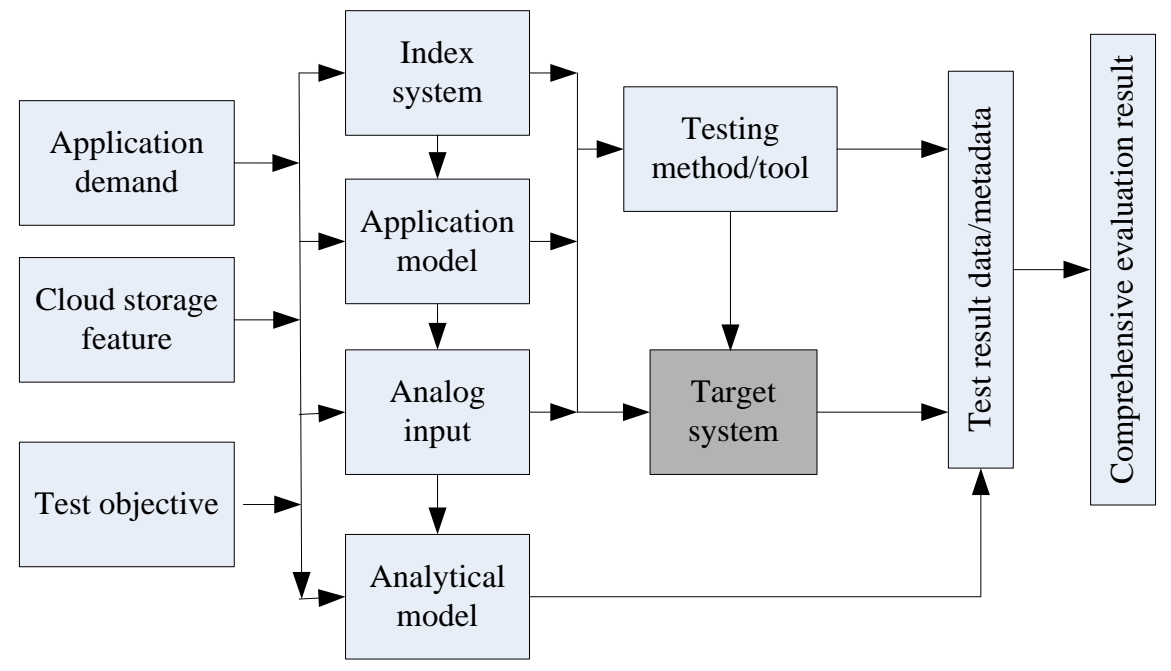

\section{Diagram 3. General Thought of Evaluation Research}

\subsection{Cloud Storage Evaluation}

Cloud storage system has multiple nodes distributed widely. Distinct difference exists for users when they use cloud storage service. Cloud storage test needs use limited and reasonable test case to complete the test of cloud storage end-user. Cloud storage environment may cause the inconsistent test result in the same test case owing to the environment differences led by time and geography, so we need study the scheme to eliminating environmental affecting factors. By virtue of the idea of testing CDN network performance with Plantnet, it can be also used for access performance test in different physical positions of distributed network environment, construct similar cloud testing environment and realize cloud testing with cloud scheme.

The paper proposes to provide a whole set of testing environment based on cloud storage platform (non-target system), so tester can carry out test instantly as long as he logs in the testing environment by means of virtual desktop. We provide software and 
hardware installation, environment configuration and environmental maintenance. After testers assign hardware configuration, software stack (operating system, middleware, tools software), network topology, it creates a new set of testing environment with current virtualization technology. If tester can accept the created standard testing environment, then he can log in immediately.

In the evaluation, "cloud testing with cloud" [7-8] is the basic testing principle. Based on this, this subject constructs testing environment and designs test standard and testing tools. The "cloud testing with cloud" program schematic is as shown in Diagram 4. Such "cloud testing with cloud" methods can not only provide complete testing environment, but also provide many additional services. For test machine, it can provide origin point for tester to reset the virtual machine to assigned state. For large-scale test, cloud test can provide multiple testing clients. They can download test case from main controller, execute and submit test result and main controller reports the result to tester after summarizing.

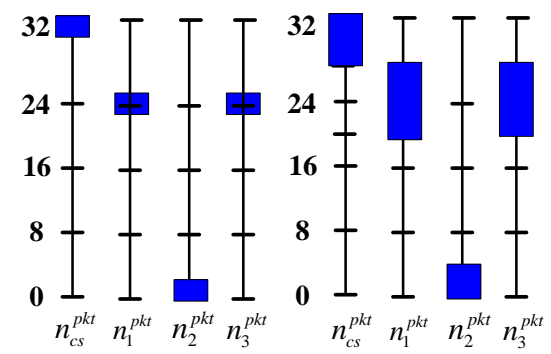

(a)

(b)

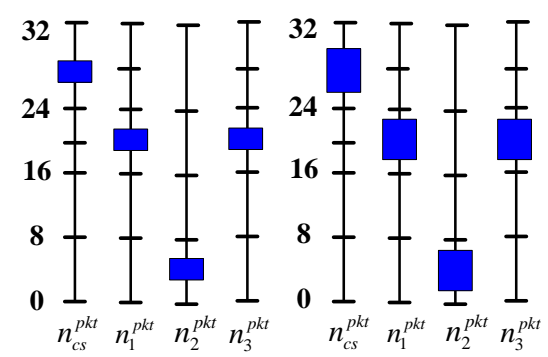

(c)

(d)

\section{Diagram 4. Storage Dynamics Step Length Algorithm Instance}

In the evaluation, "cloud testing with cloud" is the basic testing principle. Based on this, this subject constructs testing environment and designs test standard and testing tools. Testing environment and testing tool must turn into passive adapting to tested system environment from active selecting operation platform of traditional mass storage system. Therefore, the development and usage of all tools must conform to the specification of interface of cloud storage platform environment to ensure it has good transportability and compatibility and realize the target of testing cloud storage platform. Because the structural feature of cloud storage system, main tools in testing system, such as trace collection reset and fault injection tool must be used in distributed way and it must have the capability of implanting different systematic layers simultaneously with the limitation of 3D evaluation system. 


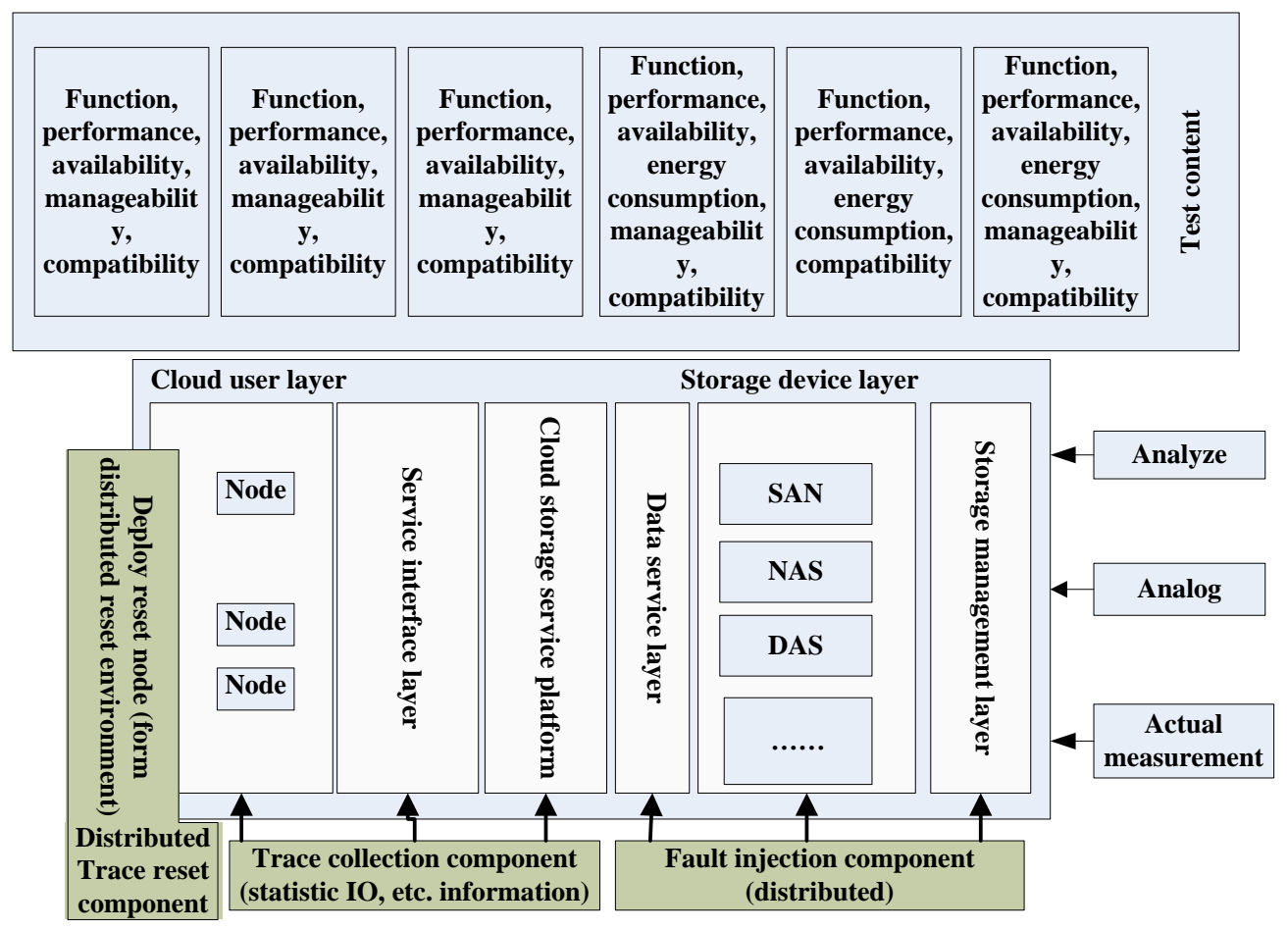

Diagram 5. Logic Diagram of Evaluation System

Cloud storage evaluation system must be combined with tested target system in logical structure closely to penetrate into the tested system, acquire effective information and evaluate system more accurately; but it should support multiple interfaces and specifications in functional realization, couple with tested system loosely, makes the scale of evaluation system can be adjusted as needed conveniently through distributed system structure so as to realize universality and flexibility of evaluation system.

As is shown in Diagram 5, the testing of target system is completed by actual measuring/ analog simulation combination analysis jointly. The evaluation will consider the whole target system, takes the black box above service interface layer as the primary thing and select different index sets in different layers for evaluation. In the dimension of user type of 3D evaluation system, it considers two modes of single user (end to end) and multiple users (distributed) according to the feature of different indexes. Trace collection tool is used in the test of performance, storage utilization, etc. Related information collection will be carried out from service platform layer to multiple layers of cloud; the most important feature of trace reset tool is to realize distributed reset.

3.4.1. Performance Index Test: Performance evaluation of cloud storage environment emphasizes on the evaluation, analysis and summarization of the whole system. In testing, it gets various performance indexes of back-end cloud storage system through front-end cloud test and computing and describes the service level provided externally by system through evaluating performance of system on various interfaces of external service.

3.4.2. Distributed Multi-Interface Trace Collection and Reset: Firstly, determine the layer of trace collection. It is carried out from external, so the grade of trace collection is closer to application. Meanwhile, the development work of evaluation system and research work proceed in parallel. In order to ensure the independence of research schedule and tool, it needs avoid strong dependency on storage system. It proposes to realize it with software package, executes trace collection in the part of system processing and responding front-end interface and ensures all captured $\mathrm{I} / \mathrm{O}$ information of system can 
record file reading, creation and retrieve, etc. According to different collection objects, Trace tool applies software and hardware realization methods. For mass information organization and access core system, it realizes it with software package, for example, seal I/O access library function on operating system. When Trace record is needed, the sealed library function can be used for recording file writing, creation, retrieve, etc., for high-end disk array, apply hardware + software method, firstly apply FC/iSCSI/FCP agreement analyzer to save all kinds of commands and results stored on network, then combine SCSI to analyze SCSI command in data package and get access demand of various data on disk array. The difficult point is the analysis of multiple agreements and paralleling time synchronization of I/O.

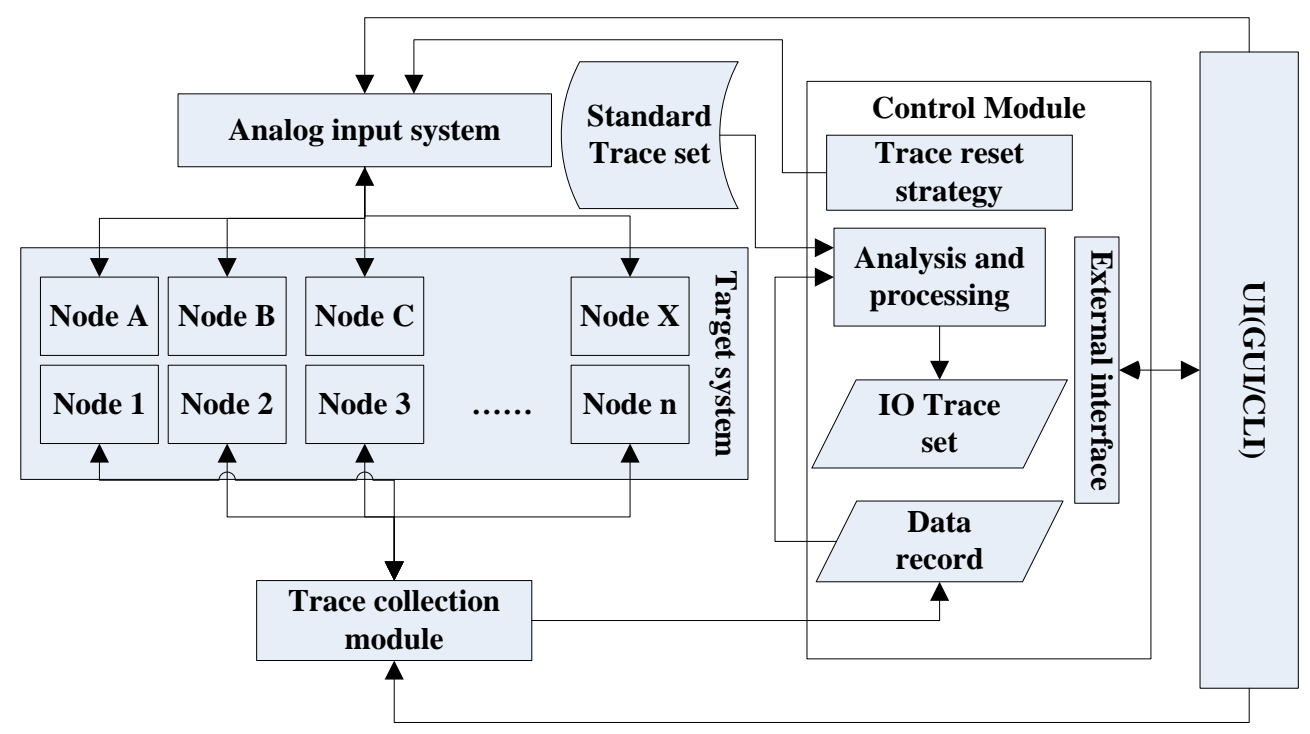

\section{Diagram 6. Trace Collection and Reset Tool Set Schematic}

Trace reset load test is usually made up of five stages: plan, scene design, erect operation environment, operation scene and result analysis. Of them, design script creation at scene design stage, reset trace set creation and scene design are as shown in Diagram 7.

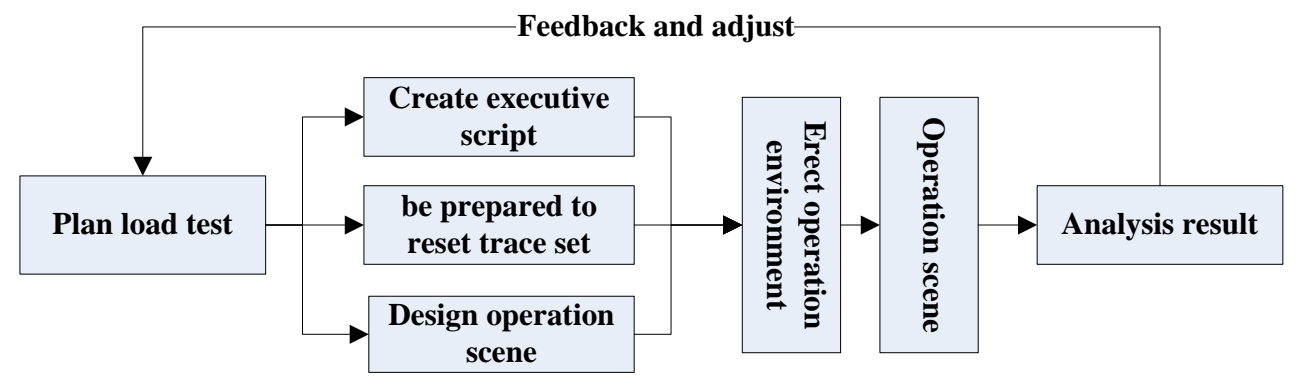

Diagram 7. Trace Reset Load Test Process

3.4.3. Expandability Test: Expandability test of cloud storage system is relatively complicated. It includes positive expansion owing to scale rising and negative expansion owing to scale decreasing. It's one of features that cloud storage system is different from mass storage system. Meanwhile, the change frequency of system sale is higher than traditional mass storage system. Therefore, in evaluation, not only does it need analyze the performance change curve of recovering stability at the time of positive expansion, but also it need analyze change curve of negative expansion. Moreover, it takes service 
state time that system recovers stability after expanding as an important evaluation index. It proposes to apply combination method of actual measuring and analyzing to carry out test on transmittability and storage capacity, etc., from different layers of application layer of cloud storage front end and cloud storage platform service layer according to $3 \mathrm{D}$ evaluation model formed by multiple dimension indexes.

Table 1. Expandability Evaluation Goal List

\begin{tabular}{|c|c|c|}
\hline Type & Content & Description \\
\hline Vertical & Expand upward & $\begin{array}{c}\text { Service ability at system node } \\
\text { unit is increased }\end{array}$ \\
\hline expandability & $\begin{array}{c}\text { Expand } \\
\text { downward }\end{array}$ & $\begin{array}{c}\text { Service ability at system node } \\
\text { unit is reduced }\end{array}$ \\
\hline Horizontal & $\begin{array}{l}\text { Expand } \\
\text { outward }\end{array}$ & $\begin{array}{l}\text { The increasing of physical } \\
\text { scale of system, such as } \\
\text { increasing of node stored } \\
\text { intelligently }\end{array}$ \\
\hline expandability & Expand inward & $\begin{array}{l}\text { The decreasing of physical } \\
\text { scale of system, such as } \\
\text { decreasing of node stored } \\
\text { intelligently }\end{array}$ \\
\hline Cost of & Cost of time & $\begin{array}{l}\text { The time cost needed by } \\
\text { system when it is expanded }\end{array}$ \\
\hline expansion & $\begin{array}{l}\text { Cost of } \\
\text { economy }\end{array}$ & $\begin{array}{l}\text { Economic cost needed by } \\
\text { system when it is expanded }\end{array}$ \\
\hline
\end{tabular}

Integrate all original data acquired through simulation and actual measuring methods, analyze data according to related model and make estimation of possible expansion scope according to actual measured data.

\section{Conclusion}

The evaluation in cloud storage environment emphasizes on evaluation, analysis and summarization of the whole situation of system. In test, it gets various performance indexes of back-end cloud storage system mainly through cloud front-end test combined with analysis and computing and describes service level provided externally by system through evaluating the performance of system in various interfaces of external service.

This paper provides a thinking and thought for solving the problem that current cloud storage system lacks purposeful comprehensive evaluation system and tool/environment. It evaluates computer infrastructure and demonstrated application developed greatly during $12^{\text {th }}$ Five-Year Plan of our country, including 0.1 billion grade parallel cloud server, EB grade cloud storage system, cloud service and management platform core software, cloud computing safe system architecture, etc. It aims at speeding up the development process of domestic computing and cloud storage product through construction promotion with evaluation and enhancing the recognition degree of domestic cloud computing and cloud storage service through unified evaluation criteria.

\section{Acknowledgement}

The research is supported by Key R \& D project of Shandong province (2015GSF120012). 


\section{References}

[1] Z. Lv, A. Halawani and S. Feng, "Multimodal hand and foot gesture interaction for handheld devices", ACM Transactions on Multimedia Computing, Communications, and Applications (TOMM), vol. 11, no 1, (2014), pp. 10.

[2] G.Liu, Y.Geng and K. Pahlavan, "Effects of calibration RFID tags on performance of inertial navigation in indoor environment", 2015 International Conference on Computing, Networking and Communications (ICNC), (2015) Febuary.

[3] J. He, Y. Geng, Y. Wan, S. Li and K. Pahlavan, "A cyber physical test-bed for virtualization of RF access environment for body sensor network”, IEEE Sensor Journal, vol. 13, no. 10, (2013) October, pp. 3826-3836.

[4] W. Huang and Y.Geng, "Identification Method of Attack Path Based on Immune Intrusion Detection", Journal of Networks, vol. 9, no. 4, (2014) January, pp. 964-971.

[5] X. Li, Z. Lv and J. Hu, "XEarth: A 3D GIS Platform for managing massive city information", Computational Intelligence and Virtual Environments for Measurement Systems and Applications (CIVEMSA), 2015 IEEE International Conference on. IEEE, (2015), pp.1-6.

[6] J. He, Y.Geng, F. Liu and C. Xu, "CC-KF: Enhanced TOA Performance in Multipath and NLOS Indoor Extreme Environment, IEEE Sensor Journal, vol. 14, no. 11, (2014) November, pp. 3766-3774.

[7] N. Lu, C. Lu, Z. Yang and Y. Geng, "Modeling Framework for Mining Lifecycle Management", Journal of Networks, vol. 9, no. 3, (2014) January, pp. 719-725.

[8] Y.Geng and K. Pahlavan, "On the accuracy of rf and image processing based hybrid localization for wireless capsule endoscopy”, IEEE Wireless Communications and Networking Conference (WCNC), (2015) March.

[9] X. Li, Z. Lv and J. Hu, "Traffic management and forecasting system based on 3d gis", Cluster, Cloud and Grid Computing (CCGrid), 2015 15th IEEE/ACM International Symposium on, (2015), pp. 991998.

[10] S. Zhang and H. Jing, "Fast log-Gabor-based nonlocal means image denoising methods", Image Processing (ICIP), 2014 IEEE International Conference on. IEEE, (2014), pp. 2724-2728.

[11] J. Hu and Z. Gao, "Distinction immune genes of hepatitis-induced heptatocellular carcinoma", Bioinformatics, vol. 28, no. 24, (2012), pp. 3191-3194.

[12] D. Jiang, Z. Xu and Z. Lv, "A multicast delivery approach with minimum energy consumption for wireless multi-hop networks", Telecommunication Systems, (2015), pp. 1-12.

[13] C. Fu, P. Zhang and J. Jiang, "A Bayesian approach for sleep and wake classification based on dynamic time warping method", Multimedia Tools and Applications, (2015), pp. 1-20.

[14] Z. Lv, "Wearable smartphone: Wearable hybrid framework for hand and foot gesture interaction on smartphone", Computer Vision Workshops (ICCVW), 2013 IEEE International Conference on. IEEE, (2013), pp. 436-443.

[15] Y. Lin, J. Yang and Z. Lv, "A Self-Assessment Stereo Capture Model Applicable to the Internet of Things", Sensors, vol. 15, no. 8, (2015), pp. 20925-20944.

[16] J. Yang, S. He and Y. Lin, "Multimedia cloud transmission and storage system based on internet of things", Multimedia Tools and Applications, (2015), pp. 1-16.

[17] Z. Lv, T. Yin and Y. Han, "WebVR——web virtual reality engine based on P2P network", Journal of Networks, vol. 6, no. 7, (2011), pp. 990-998.

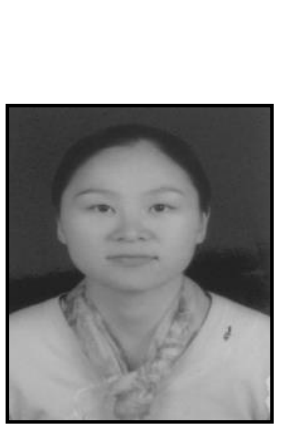

\section{Authors}

LI YongHong, received her MS degree in software engineering from Shandong university, Shandong ,China. She is currently working as IT engineer in the Earthquake Administration of Shandong Province. Her research interest is mainly in the area of big data processing and cloud computing. She has published several research papers in academic journals in the research areas mentioned above and has participated in compiling several books. 
International Journal of Grid and Distributed Computing Vol. 9, No. 10 (2016) 